\title{
Bandwidth Enhancement on Waveguide Transition to Conductor Backed CPW With High Dielectric Constant Substrate
}

\author{
Cheng-Fu Hung, An-Shyi Liu, Chih-Hung Chien, Chun-Long Wang, and Ruey-Beei Wu
}

\begin{abstract}
This paper proposes a broadband transition design between conductor-backed coplanar waveguide (CB-CPW) and rectangular waveguide based on the concept of the multisection transformer. The genetic algorithm (GA) was adopted to efficiently optimize the multisection transformer for achieving wideband performance. Using a multisection of order 3, a V-band transition realized on an $\mathrm{Al}_{2} \mathrm{O}_{3}$ substrate could achieve $25 \%$ bandwidth with a return loss better than $20 \mathrm{~dB}$. Two transitions connected back to back were realized to demonstrate the feasibility of the transition. Also, a tolerance analysis was performed via the simulation to verify the robustness of this transition design.
\end{abstract}

Index Terms-Conductor backed coplanar waveguide (CB-CPW), genetic algorithm (GA), multisection transformer, waveguide transition.

\section{INTRODUCTION}

$\mathbf{P}$ LANAR transmission lines are prevailing in the microwave and millimeter-wave frequencies due to the advances of planar integrated technology. Among them, coplanar waveguide (CPW) gradually becomes popular due to its lower dispersion than microstrip and easier integration with series and shunt devices. The conductor-backed CPW (CB-CPW), a variant of $\mathrm{CPW}$, is a better choice because it provides the additional benefits of added mechanical support and heat sinking ability compared to conventional CPW. Nonetheless, metallic waveguides still play an essential role in high power components like antenna feeds, high $Q$ filters, diplexers, polarizers, and so on. Thus, it is interesting to design transitions between CB-CPW and rectangular waveguide when integrating different circuit components.

Transition design between rectangular waveguide and planar transmission lines has been presented in 1993 [1]. Recently, a transition using a uniplanar quasi-Yagi antenna on a high permittivity substrate [2] was reported. This design is easily integrated; however, the bandwidth is limited by the Yagi array. In addition, the structure would be too large to be inserted in the waveguide for low permittivity design.

To overcome this problem, a unified transition between rectangular waveguide and various planar transmission lines has

\footnotetext{
Manuscript received May 26, 2004; revised November 1, 2004. This work was supported in part by the National Science Council of Taiwan, R.O.C., under Grant NSC91-2219-E002-017, by the Ministry of Education under Grant MoE89-E-FA-06-2, and by Airwave Inc. The review of this letter was arranged by Associate Editor A. Stelzer.

The authors are with the Department of Electrical Engineering and the Graduate Institute of Communication Engineering, National Taiwan University, Taipei 106, Taiwan, R.O.C. (e-mail: r91942003@ntu.edu.tw).

Digital Object Identifier 10.1109/LMWC.2004.842862
}

been proposed [3], based on the concept of tapered coplanar strip (TCPS) antenna and one section of impedance transformer. Although the transition could be realized no matter that the permittivity of the substrate is low or high, the transition being designed on the high permittivity substrate such as $\mathrm{Al}_{2} \mathrm{O}_{3}\left(\varepsilon_{r}=9.9\right)$ has limited bandwidth [4]. To solve this problem, this paper proposes a multisection transformer for the wideband-matching and employs the genetic algorithm [5] for the optimal dimensions.

\section{DESCRIPTION OF TRANSITION}

The CB-CPW to rectangular waveguide transition under investigation is shown in Fig. 1. It is composed of three parts: a CB-CPW to slotline transition on the left hand side, a TCPS antenna inserted into the E-plane of the end launcher of the waveguide on the right hand side, and a multisection slotline transformer used to match the two parts mentioned above for wideband performance.

For the CB-CPW to slotline transition, the CB-CPW mode is converted to the slotline mode through the two slotlines with $180^{\circ}$ phases-difference. Such transition could enhance a wide band performance [6]. To suppress the odd mode and parallel plate mode of the CB-CPW, vias between the upper and lower ground planes are used. Note that these vias take the place of air-bridges that are typically used to suppress the odd mode.

For the TCPS to waveguide transition, the energy on the TCPS could easily be transmitted to the waveguide since the field pattern of the antenna has a good match to the dominant $\mathrm{TE}_{10}$ mode of the rectangular waveguide [7]. The length of TCPS is about one quarter free-space wavelength for achieving resonance at which the radiation to waveguide is efficient as shown in Fig. 1(b). The metal edge of the TCPS is kept isolated from the metallic walls of waveguide. This can ease the fabrication and improve the reliability since the TCPS can be directly inserted into an ordinary waveguide and soldering is unnecessary.

In between is the multisection slotline transformer to match the afore-mentioned two sub-transitions. To facilitate the design, genetic algorithm (GA) [5] is used here to optimize for wideband transition performance. The two sub-transitions were simulated by the full-wave field solver Ansoft HFSS. During the optimization, they are substituted by the simulated S matrices. and the multisection slotline transformer is modeled by cascaded transmission lines. Thus, the overall performance of the entire transition shown in Fig. 1 can be calculated very efficiently and the GA optimization becomes feasible. The major 


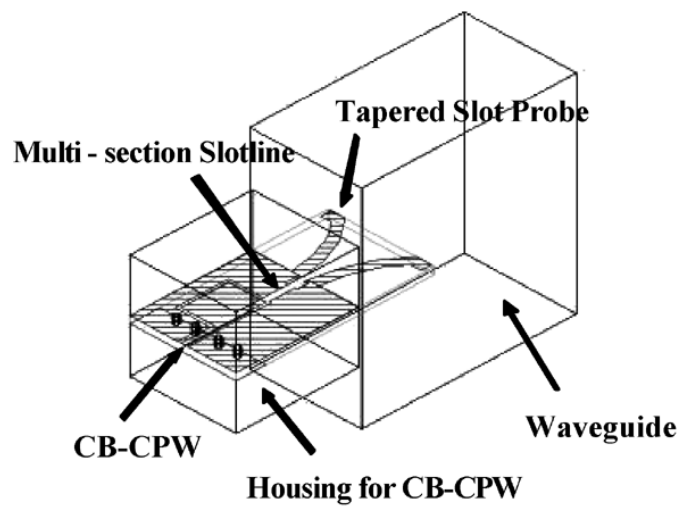

(a)

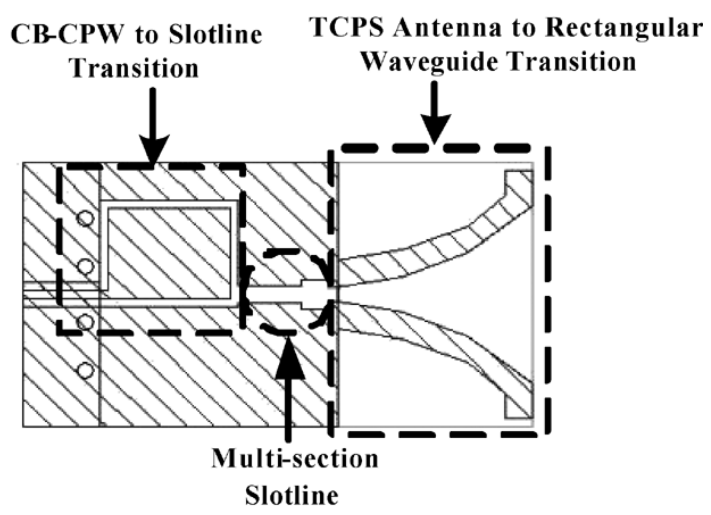

(b)

Fig. 1. Structure of CB-CPW to rectangular waveguide transition (a) 3-D view and (b) upper view.

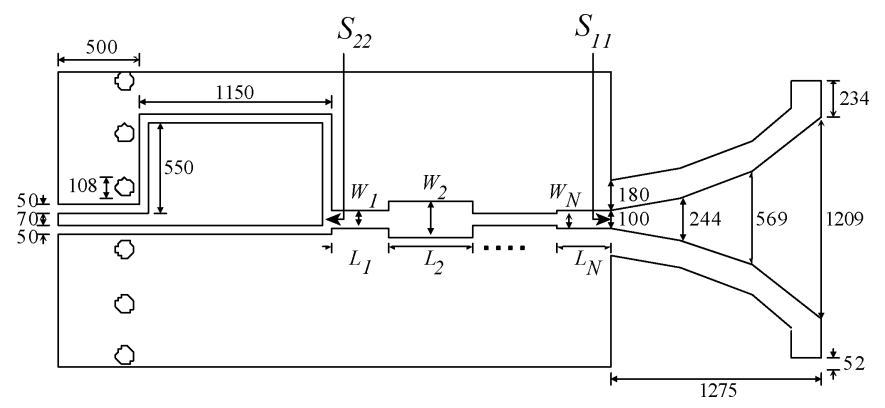

Fig. 2. Top view of conductor layout with its dimensions marked.

TABLE I

WIDTHS $W_{N}$ AND LENGTHS $L_{N}$ FOR VARIOUS ORDERS OF MULTI-SECTION TRANSFORMER

\begin{tabular}{c|c|c|c|c|c|c}
\hline $\mathrm{N}$ & $W_{l}$ & $L_{l}$ & $W_{2}$ & $L_{2}$ & $W_{3}$ & $L_{3}$ \\
\hline 1 & 140 & 844 & $\mathrm{X}$ & $\mathrm{X}$ & $\mathrm{X}$ & $\mathrm{X}$ \\
\hline 2 & 203 & 508 & 178 & 508 & $\mathrm{X}$ & $\mathrm{X}$ \\
\hline 3 & 110 & 411 & 200 & 174 & 100 & 61 \\
\hline
\end{tabular}

advantage of this optimization approach is that no optimal design of the two sub-transitions is required. And the wideband performance could be achieved by combining the multisection transformer and GA.

\section{Simulation AND MEASUREMENT Results}

To realize the V-band transition, a standard WR-15 waveguide with inside dimension $148 \times 74 \mathrm{mil}^{2}$ is used as the transi-

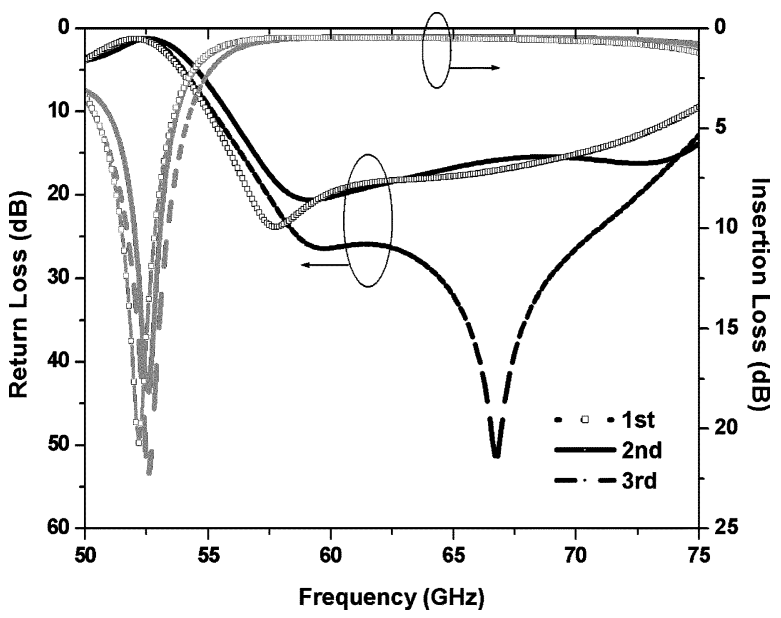

Fig. 3. Simulated insertion and return losses versus frequency for the structure with multisection transformers of orders 1,2 , and 3 .

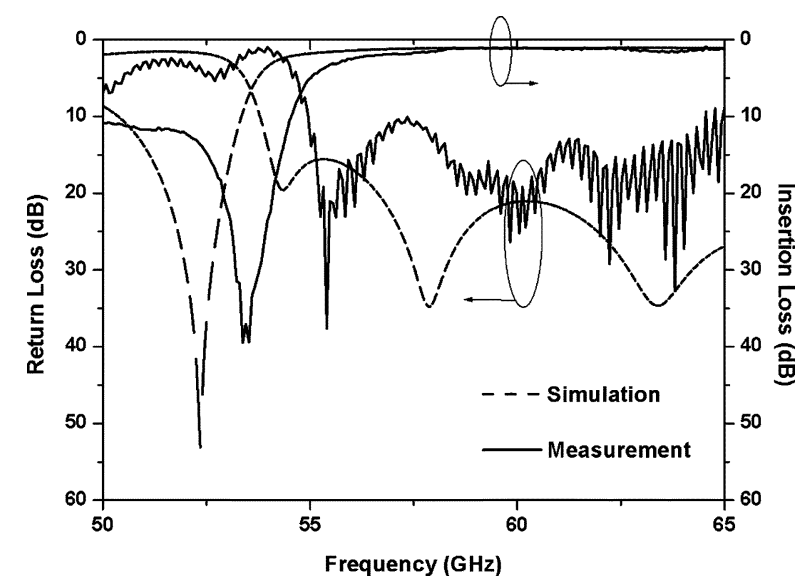

Fig. 4. Comparison between simulation and measurement results for the structure in Fig. 4.

tion waveguide, while $\mathrm{Al}_{2} \mathrm{O}_{3}$ substrate with 5-mil-thickness and dielectric constant of 9.9 is chosen to implement the planar circuit as shown in Fig. 1(a). In the structure, the lower conductor plane of the CB-CPW is placed at the center of the housing with a housing size of $70 \times 74 \mathrm{mil}^{2}$ and is in contact with the metal wall of the housing. The diameter of vias is $5 \mathrm{mil}$, and the gap between vias is at least 2 mil away from CPW slots since it should be smaller than half guided wavelength to avoid parallel plate mode.

To accomplish the transition, first, the CB-CPW to slotline and TCPS antenna to rectangular waveguide transitions having the dimensions in micrometer indicated in Fig. 2 are simulated by the commercial software Ansoft HFSS. After that, the dimensions of the multisection are determined by using the genetic algorithm (GA) on the basis of minimizing the root-mean-squares of $\left|S_{11}\right|$ in the frequency range from 55 to $75 \mathrm{GHz}$.

The GA optimized dimensions of the multisection matching circuits are shown in Table I for one-, two-, and three-section transformers. One thing worth mentioning is that the discontinuity effects between two sections are neglected while the widths and lengths of the sections are changed in the optimization procedure. Nonetheless, the optimized structures are put into HFSS for full-wave analysis and the simulation results are 


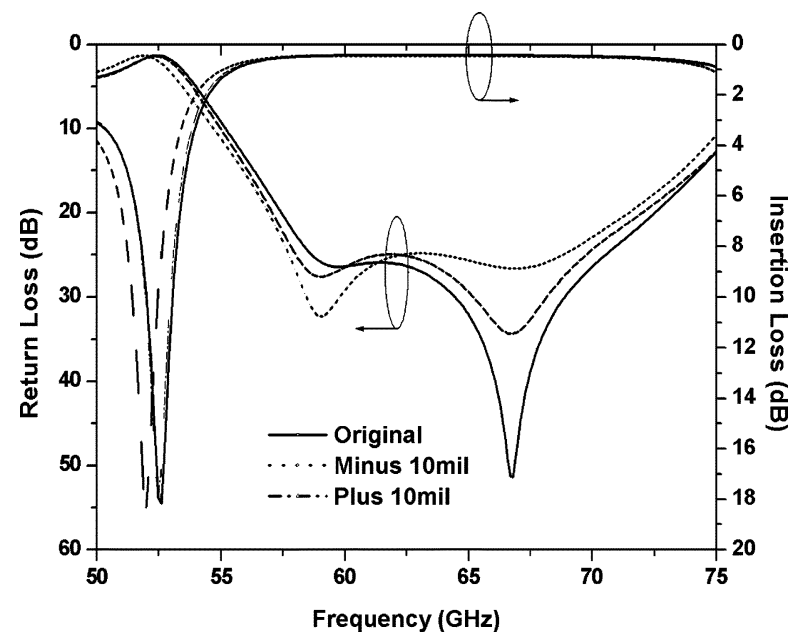

Fig. 5. Simulation results with different substrate vertical position as a parameter.

shown in Fig. 3. The insertion and return losses for the transitions using one-, two-, and three-section transformers are compared. It can be found that the return loss for the case of threesection can reach $25 \%$ bandwidth in which the return loss is better than $20 \mathrm{~dB}$.

For experimental verification, the designed transitions with three-section transformer are fabricated and connected in a back-to-back configuration as shown in the inset of Fig. 4. The comparisons of simulation and measurement results are shown in Fig. 4. It can be seen that the simulation and measurement exhibit similar tendency but have some frequency shift. The discrepancy may be contributed to the limited fabrication resolution or the imperfect measurement calibration in such a high frequency band. Although the return loss seems to have a large deviation, the measured results are still better than $10 \mathrm{~dB}$ above $55 \mathrm{GHz}$. In general, the insertion losses of simulated and measured results match well in the passband.

\section{TOLERANCE ANALYSIS}

The above simulation and measurement results prove that CPW to rectangular waveguide transitions can easily achieve broadband and low-loss performance. However, the mechanical sensitivity of this design should still be checked. Here, the tolerance of mechanical assembly and alignment of the transition are verified by the position offset of the circuit and the width of the substrate.

Fig. 5 shows the simulation results of this transition when the substrate is positioned \pm 10 -mil offset vertically from the center of CB-CPW housing shown in Fig. 1. Although the offset we assumed is relatively large $( \pm 13.5 \%)$, the changes in simulation are not obvious. The bandwidth (return loss better than $20 \mathrm{~dB}$ ) of the two cases is almost the same.

Fig. 6 shows the simulation results of this transition when the substrate width is 2 and 4 mil shorter than the original one $((W=70 \mathrm{mil})$. The other structure parameters are kept the same. The results show that the bandwidth is reduced significantly when the width is 4 mil shorter (66 mil). However, the substrate truncation error is quite small in modern technologies; this error can be substantially reduced.

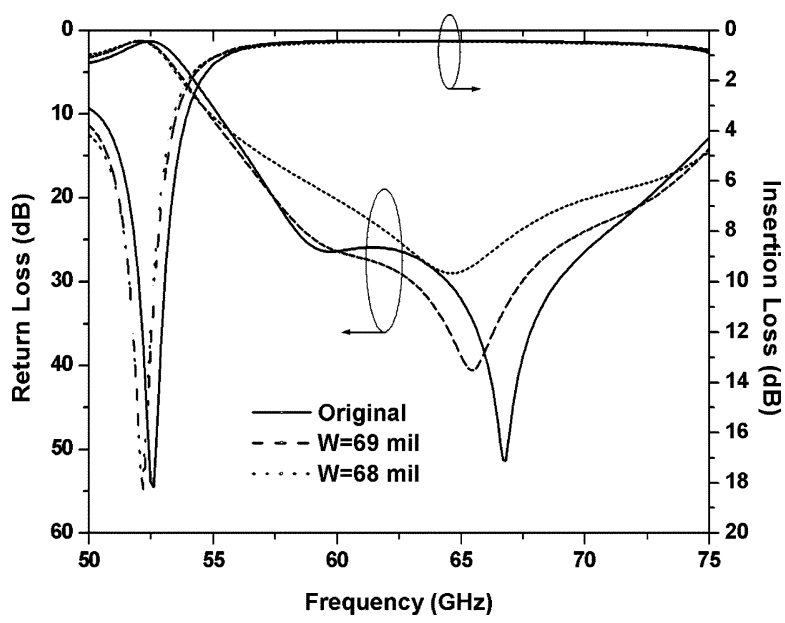

Fig. 6. Simulation results with different substrate width as a parameter.

\section{CONClusion}

A conductor backed CPW (CB-CPW) to waveguide transition is developed based on the concept of tapered slot antennas and GA optimized multi-section transformers. A V-band transition using single section transformer could only achieve $5 \%$ bandwidth in which the return loss is better than $20 \mathrm{~dB}$. By using the three-section transformer, the full-wave simulation of the V-band transition demonstrates $25 \%$ bandwidth.

To verify the design, a back-to-back transition is realized and measured to demonstrate the feasibility of this structure. Reasonable agreement can be found from the comparison between the simulation and measurement results, in view of the difficulty in the fabrication resolution control and measurement calibration at such a high frequency band.

The tolerance analysis is also addressed and it shows that the transition is robust in vertical alignment error and substrate truncation error. The optimized transition is shown to operate well over almost the entire recommended waveguide band exhibiting the advantages of broad bandwidth, compact size, low fabrication cost, and high reliability.

\section{REFERENCES}

[1] J. Machac and W. Mentzel, "On the design of waveguide to microstrip and coplanar line transitions," in Proc. 23rd Eur. Microwave Conf., Madrid, Spain, 1993, pp. 615-616.

[2] N. Kaneda, Y. Qian, and T. Itoh, "A broadband CPW-to-waveguide transition using quasi-Yagi antenna," in IEEE MTT-S Int. Dig., vol. 2, Boston, MA, Jun. 2000, pp. 617-620.

[3] T. H. Lin and R. B. Wu, "CPW to waveguide transition with tapered slotline probe," IEEE Microw. Wireless Compon. Lett., vol. 11, no. 7, pp. 314-316, Jul. 2001.

[4] - , "A broadband microstrip-to-waveguide transition with tapered CPS probe," in Proc. 32nd Eur. Microwave Conf., Milan, Italy, Sep. 2002.

[5] M. Gen and R. Cheng, Genetic Algorithms and Engineering Design. New York: Wiley-Interscience, 1997, pp. 7-31.

[6] K. P. Ma, Y. Qian, and T. Itoh, "Analysis and applications of a new CPWslotline transition," IEEE Trans. Microw. Theory Tech., vol. 47, no. 4, pp. 426-432, Apr. 1999.

[7] T. H. Lin, "FEM accuracy improvement and waveguide transition design," PhD. dissertation, Nat. Taiwan Univ., Taipei, Taiwan, R.O.C., Jun. 2001. 